\title{
Effects of Mirror Therapy Using a Tablet PC on Central Facial Paresis in Stroke Patients
}

\author{
Jung-A Kang, MD, Min Ho Chun, MD, PhD, Su Jin Choi, MD, \\ Min Cheol Chang, MD, You Gyoung Yi, MD
}

Department of Rehabilitation Medicine, Asan Medical Center, University of Ulsan College of Medicine, Seoul, Korea

\begin{abstract}
Objective To investigate the effects of mirror therapy using a tablet PC for post-stroke central facial paresis. Methods A prospective, randomized controlled study was performed. Twenty-one post-stroke patients were enrolled. All patients performed 15 minutes of orofacial exercise twice daily for 14 days. The mirror group $(n=10)$ underwent mirror therapy using a tablet PC while exercising, whereas the control group ( $\mathrm{n}=11)$ did not. All patients were evaluated using the Regional House-Brackmann Grading Scale (R-HBGS), and the length between the corner of the mouth and the ipsilateral earlobe during rest and smiling before and after therapy were measured bilaterally. We calculated facial movement by subtracting the smile length from resting length. Differences and ratios between bilateral sides of facial movement were evaluated as the final outcome measure.

Results Baseline characteristics were similar for the two groups. There were no differences in the scores for the basal Modified Barthel Index, the Korean version of Mini-Mental State Examination, National Institutes of Health Stroke Scale, R-HBGS, and bilateral differences and ratios of facial movements. The R-HBGS as well as the bilateral differences and ratios of facial movement showed significant improvement after therapy in both groups. The degree of improvement of facial movement was significantly larger in the mirror group than in the control group. Conclusion Mirror therapy using a tablet PC might be an effective tool for treating central facial paresis after stroke.
\end{abstract}

Keywords Facial paresis, Mirror therapy, Stroke

\section{INTRODUCTION}

Facial paresis is a very common post-stroke sequela, and it leads to functional and esthetic defects in patients [1]. Facial paresis results in drooping of the corners of the mouth, salivary drooling, spilling of food during meals, asymmetric smiles, and unclear speech [2]. This complication not only makes a stroke patients' daily living inconvenient but also discourages them from having a social life. Consequently, facial paresis could affect the

Received August 11, 2016; Accepted October 3, 2016

Corresponding author: Min Ho Chun

Department of Rehabilitation Medicine, Asan Medical Center, University of Ulsan College of Medicine, 88 Olympic-ro 43-gil, Songpa-gu, Seoul 05505, Korea. Tel: +82-2-3010-3800, Fax:+82-2-3010-6964, E-mail: mhchun@amc.seoul.kr

ORCID: Jung-A Kang (http://orcid.org/0000-0001-5275-278X); Min Ho Chun (http://orcid.org/0000-0001-8666-7225); Su Jin Choi (http://orcid. org/0000-0002-3443-2237); Min Cheol Chang (http://orcid.org/0000-0002-7629-7213); You Gyoung Yi (http://orcid.org/0000-0003-1791-1831).

(c) This is an open-access article distributed under the terms of the Creative Commons Attribution Non-Commercial License (http://creativecommons.org/ licenses/by-nc/4.0) which permits unrestricted noncommercial use, distribution, and reproduction in any medium, provided the original work is properly cited. Copyright ( 2017 by Korean Academy of Rehabilitation Medicine 
patients' psychosocial well-being [3]. Konecny et al. [4] showed that there is a very close relationship between changes in facial paresis and Beck Depression InventoryII scores (BDI-II), as well as a correlation between the House-Brackmann Grading Scale (HBGS) and the overall quality of life. Thus, management of facial paresis after a stroke is an important aspect of post-stroke rehabilitation.

Generally, orofacial exercises are used to rehabilitate facial paresis after a stroke, but few studies have proven the effectiveness of such exercises [2,5]. Moreover, no other effective therapies have yet been established.

However, in peripheral-type facial palsy, more varied treatments are being employed. One is mirror therapy. Nakamura et al. [6] used mirror therapy to prevent synkinesis after facial palsy. Azuma et al. [7] also used mirror therapy with single-dose botulinum toxin for the treatment of facial synkinesis after Bell's palsy and herpes zoster. These two studies found a significant beneficial effect of mirror therapy. In these studies, mirror therapy was conducted by simply watching the mirror while exercising. Thus, authors postulated an underlying biofeedback mechanism.

The effect of mirror therapy in stroke patients may be different from that in peripheral-type facial palsy. Neural plasticity is considered to be a main mechanism of mirror therapy in stroke patients, and it is generally considered to be mediated by mirror-induced illusion [8]. Mirror therapy has been conventionally used in the Department of Stroke Rehabilitation to treat upper-limb and lowerlimb weakness [9]. In 2012, a Cochrane Review of 14 studies, which included 567 patients after stroke, found a positive effect of mirror therapy on motor function and activities of daily living [10]. To date, there has been no precedent in the rehabilitation of post-stroke central facial paresis using mirror-induced illusion.

The aim of this study was to evaluate the effect of mirror therapy on central facial paresis after stroke. To make illusional movements of the face, we used a tablet PC mirror application during orofacial exercise, expecting to find a new rehabilitation method effective for post-stroke central facial paresis.

\section{MATERIALS AND METHODS}

\section{Participants}

We enrolled 21 stroke patients with central facial paresis in the sub-acute post-stroke phase between November 1, 2014, and December 31, 2015. Patients meeting the following criteria were recruited into the study: (1) diagnosed with first unilateral hemispheric stroke confirmed by neuroimaging examinations, computed tomography (CT), and/or magnetic resonance imaging (MRI); (2) transferred to the Department of Rehabilitation Medicine within 12 weeks of stroke onset; and (3) able to understand the study design, as screened by the ability to follow a three-step command.

We excluded the following patients: (1) those regarded as peripheral facial palsy: without forehead movement, and/or lesion at facial motor nucleus in CT and/or MRI; (2) those with visual disturbances, or those who could not distinguish their face reflected in the mirror, or had hemispatial neglect; (3) those with a history of facial palsy of the central type and/or peripheral type; and (4) those diagnosed with total paralysis from stroke onset, having no ability to move the mouth.

This study was approved by Asan Medical Center Institutional Review Board (No. S2014-0995-0002), and written informed consent was obtained from each patient before study initiation.

\section{Treatment}

We randomly divided patients into two groups via computer-generated randomization code: 10 in the mirror group, 11 in the control group. Initially, all patients received lessons on orofacial exercises from a speech and language therapist. They performed the exercise for 15 minutes two times a day for 14 days. We used a tablet PC mirror application that can convert images from right to left. In the mirror group, the mirror application was used during the exercise. Patients watched the tablet PC screen. The mirror application converts the image from right to left and then applies a shade over the half of the screen opposite to the unaffected side (Fig. 1). As a result, patients watched the reflection of the unaffected half of the face as if it were the affected face.

\section{Outcomes}

All patients' medical data, including age, sex, and type 


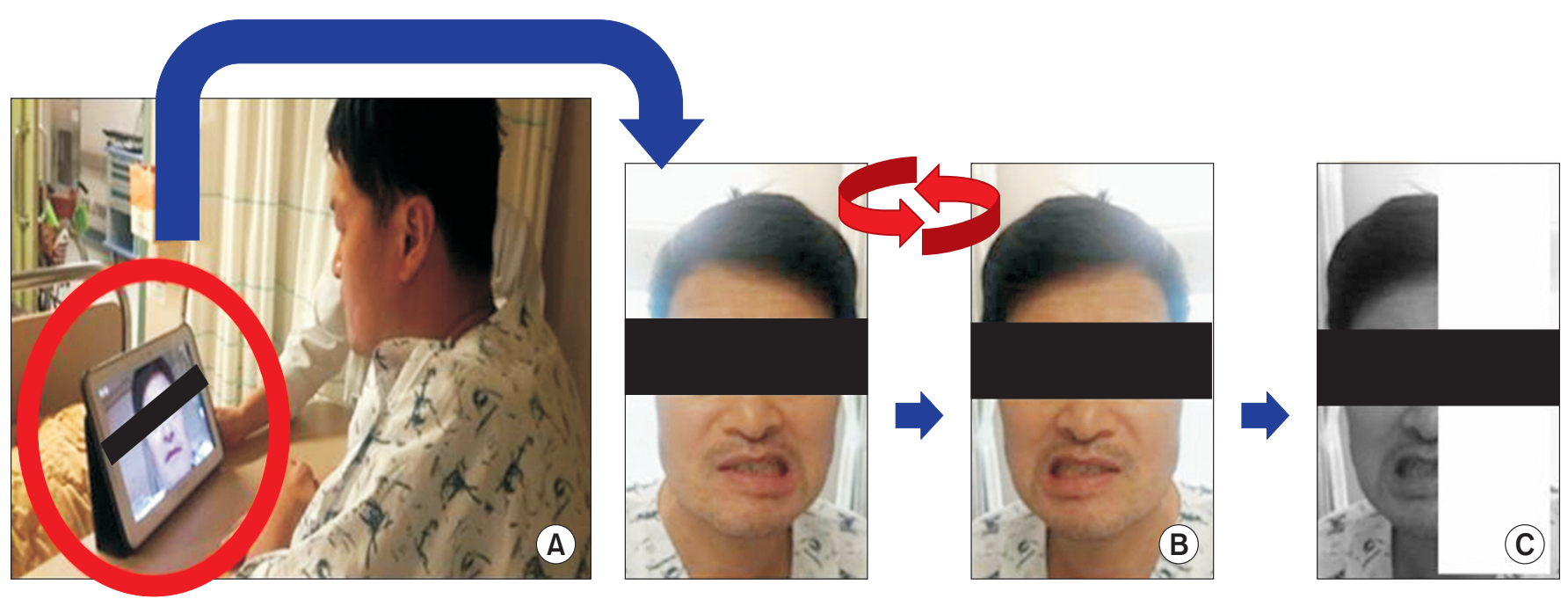

Fig. 1. (A) Patients watch the tablet PC screen. (B) Apply mirror application; convert the right and left side of the screen. (C) Shade the half of the screen which is opposite the unaffected side. As a result, patients watch the reflection of the unaffected half of the face as if it were the affected half.

of stroke (ischemic or hemorrhagic), were reviewed. Basal Modified Barthel Index (MBI), Korean version of Mini-Mental State Examination (K-MMSE), and National Institutes of Health Stroke Scale (NIHSS) were used to assess for baseline stroke severity. We assessed the degree of facial paresis before and after the treatment using the following tools.

\section{Regional House-Brackmann Grading System}

R-HBGS is a modification of the HBGS. We evaluated movement of the mid-face and mouth, because forehead movement is preserved in central facial palsy. Patients were scored as follows: 1 , normal movement; 2 , slight weakness; 3 , obvious but not disfiguring weakness, symmetric at rest; 4 , obvious weakness and disfiguring asymmetry with motion, symmetric at rest; 5 , barely perceptible motion in the mid-face, asymmetric at rest; 6 , no movement [11].

\section{Length between the corner of the mouth and the earlobe}

We measured the length between the corner of the mouth and the ipsilateral earlobe during rest $\left(L_{\text {rest }}\right)$ and smile $\left(L_{\text {smile }}\right)$. Modifying the study of Konecny et al. [2], we used measuring tape directly on the patients' face. Then the facial movement $(M)$ was calculated as $L_{\text {rest }}$ $L_{\text {smile }}$. The facial movement was compared between the two sides. First, movement differences (M-dif) between
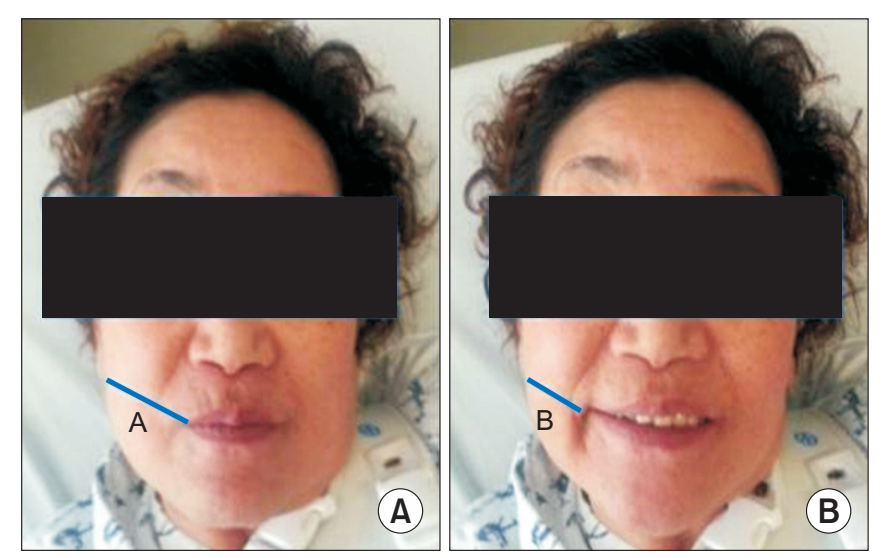

Fig. 2. (A) Length at resting $\left(L_{\text {rest }}\right)$ and (B) length at smiling $\left(L_{\text {smile }}\right)$. Movement $(\mathrm{M})=\mathrm{L}_{\text {rest }}-\mathrm{L}_{\text {smile }}$. Movement difference $(M-$ dif $)=[M($ nonparetic $)]-[M($ paretic $)]$. Movement ratio $(M-r a t)=[M($ paretic $)] /[M($ nonparetic $)]$.

the sides was calculated by subtracting the paretic-side $M$ from the nonparetic-side $M$. Second, the movement ratio ( $M$-rat) of the paretic-side $M$ to that of the nonpareticside $M$ was also calculated (Fig. 2). These outcomes were measured at baseline and after the study. Two physiatrists evaluated the outcomes three times, and the mean value was used for analysis.

\section{Statistical analysis}

Differences in the study population were analyzed by the t-test or the Mann-Whitney U test. Wilcoxon signed- 
rank test was used to evaluate the effect of therapy in both groups. Comparison of the effectiveness of the therapy for the two groups was analyzed using the MannWhitney $\mathrm{U}$ test. The $\mathrm{p}$-values of $<0.05$ were considered to indicate a statistical significance. This statistical analysis was conducted with SPSS ver. 19.0 (IBM, Armonk, NY, USA).

\section{RESULTS}

Twenty-one patients, including 13 men and 8 women, were assessed. Mean ages were 63.1 and 55.6 years in the mirror and control groups, respectively. The baseline stroke degree, including basal MBI, K-MMSE, and NIHSS scores, was similar between the groups. Baseline facial paresis was evaluated by R-HBGS, $M$-dif, and $M$-rat; the scores were not significantly different between the two groups (Table 1).

All measurements showed significant differences between baseline and after therapy in both groups (Fig. 3). In the mirror group, the R-HBGS scores in the mid-face were $2.9 \pm 0.7$ and $2.1 \pm 1.0$ at baseline and after therapy, respectively. In the mouth, the R-HBGS score changed from 3.3 \pm 1.6 to $2.3 \pm 1.6$. $M$-dif decreased from a baseline of 3.5 \pm 1.9 to $2.0 \pm 1.8$ after therapy. $M$-rat increased from a baseline of $0.5 \pm 0.2$ to $0.7 \pm 0.2$ after therapy. In the control group, R-HBGS in the mid-face changed from $2.5 \pm 0.5$ to $2.1 \pm 0.7$, whereas that in the mouth changed from $3.5 \pm 1.1$ to $2.8 \pm 1.3$. $M$-dif decreased from $3.9 \pm 2.0$ to $3.4 \pm 2.0$ after therapy. $M$-rat increased from $0.5 \pm 0.2$ to $0.6 \pm 0.2$ after therapy.

Table 2 compares the two groups. The improvement in facial movement, which is measured by the $M$-dif, was significantly larger in the mirror group $(\mathrm{p}=0.04)$. The mean difference was $1.45 \pm 0.90$ in the mirror group and $0.55 \pm 1.00$ in the control group. The mean change of $M$ rat was also larger in the mirror group, at $0.30 \pm 0.19$, than that in the control group, at $0.11 \pm 0.12(\mathrm{p}=0.01)$.

\section{DISCUSSION}

Compared with the control group, the group receiving the orofacial exercise with the use of the tablet PC mirror application showed greater improvement in facial movement after stroke.

In stroke rehabilitation, mirror therapy has been demonstrated to improve the patient's motor function, especially of the upper limbs. Selvaraj et al. [12] studied 20 patients with middle cerebral artery infarction within six months after stroke onset. The study group received an additional hour of mirror therapy for 3 weeks. They found significantly greater improvements in Fugl-Meyer scores, Brunnstrom stages, and Box and Block Test scores in the study group than in the control group [13]. Recently, mirror therapy was studied in chronic stroke patients. Colomer et al. [14] conducted a randomized controlled trial including 31 patients enrolled $>6$ months after stroke. They found improvement in upper-limb motor function

Table 1. Baseline characteristics of patients

\begin{tabular}{lccc}
\hline & Mirror group $(\mathbf{n}=\mathbf{1 0})$ & Control group $(\mathbf{n}=\mathbf{1 1})$ & p-value \\
\hline Sex (male:female) & $6: 4$ & $7: 4$ & 0.22 \\
\hline Age & $63.1 \pm 10.3$ & $55.6 \pm 16.0$ & 0.83 \\
\hline Stroke type (ischemic:hemorrhagic) & $9: 1$ & $11: 0$ & 0.48 \\
\hline K-MMSE & $22.8 \pm 4.2$ & $25.6 \pm 4.4$ & 0.15 \\
MBI & $40.1 \pm 25.9$ & $45.6 \pm 29.2$ & 0.65 \\
NIHSS & $10.6 \pm 4.2$ & $10.0 \pm 5.0$ & 0.83 \\
\hline HBGS & $3.2 \pm 1.2$ & $3.4 \pm 1.1$ & 0.22 \\
\hline Movement difference $(\mathrm{mm})$ & $3.5 \pm 1.9$ & $3.9 \pm 2.0$ & 0.61 \\
\hline Movement ratio & $0.5 \pm 0.2$ & $0.5 \pm 0.2$ & 0.89 \\
\hline
\end{tabular}

Values are presented as number or mean \pm standard deviation.

K-MMSE, Korean version of Mini-Mental State Examination; MBI, Modified Barthel Index; NIHSS, National Institutes of Health Stroke Scale; HBGS, Regional House-Brackmann facial nerve Grading System; movement difference, difference of facial movement between sides; movement ratio, ratio of facial movement between sides.

${ }^{*} \mathrm{p}<0.05$ by Mann-Whitney $\mathrm{U}$ test. 
(A)

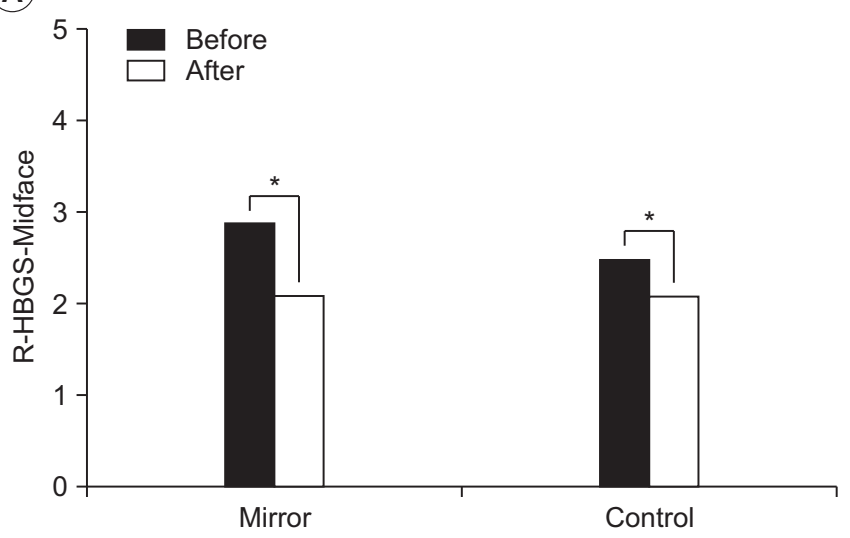

(C)

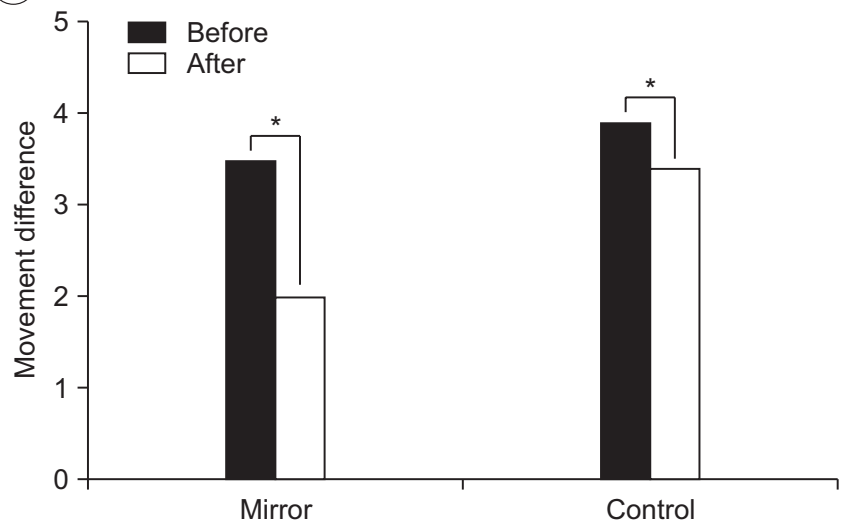

(B)

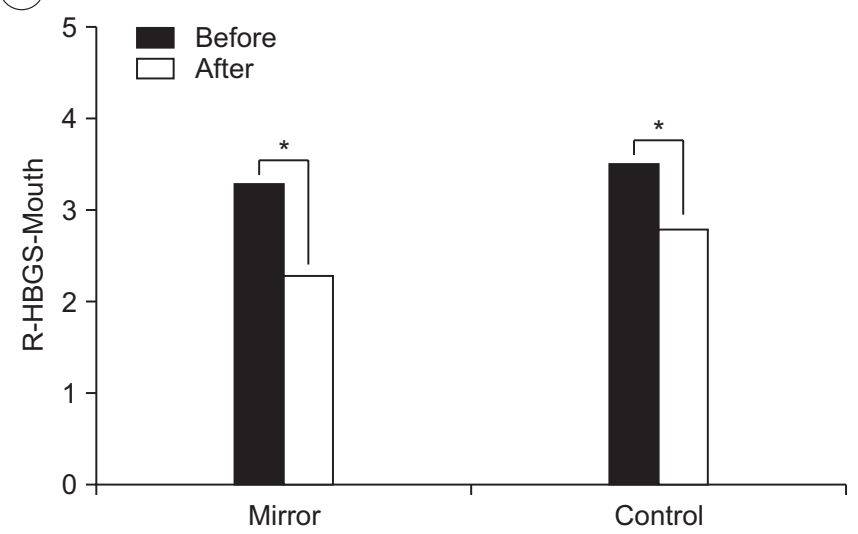

(D)

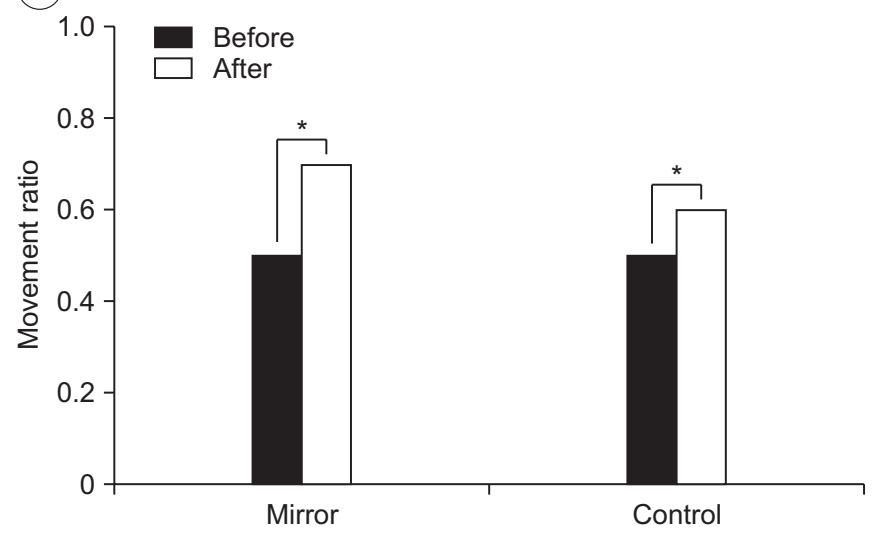

Fig. 3. Comparison of the differences before and after treatment in the mirror group $(n=10)$ and control group $(n=11)$. R-HBGS, Regional House-Brackmann facial nerve Grading System. ${ }^{*} \mathrm{p}<0.05$ by Wilcoxon signed-rank test.

Table 2. Degree of improvement of facial movement

\begin{tabular}{lccc}
\hline & Mirror therapy & Conventional therapy & p-value \\
\hline$\Delta$ Movement difference $(\mathrm{mm})$ & $1.45 \pm 0.90$ & $0.55 \pm 1.00$ & $0.04^{*}$ \\
$\Delta$ Movement ratio & $0.30 \pm 0.19$ & $0.11 \pm 0.12$ & $0.01^{*}$ \\
\hline
\end{tabular}

Values are presented as mean \pm standard deviation.

${ }^{*} \mathrm{p}<0.05$ by Mann-Whitney U test.

and tactile sensation. Mirror therapy was also demonstrated to improve lower-limb motor function. Sutbeyaz et al. [15] studied 40 patients without volitional ankle dorsiflexion within 12 months after stroke onset. After 4 weeks of additional 30-minute daily mirror therapy, Brunnstrom stages and Functional Independence Measure motor scores showed improvements in the study group.

The mechanism of the effect of mirror therapy is considered to be through neural plasticity, which is the principal concept of recovery in stroke patients. It refers to the capacity and resiliency of the brain to change structure and function, enabling the brain to adapt to different conditions after injury. To reveal the impact of mirror therapy on neural plasticity, several studies were conducted with neuroimaging. Guo et al. [16] used functional magnetic resonance imaging (fMRI) to study mirror-induced ankle movements. They investigated five stroke patients performing contralateral ankle flexion/ extension while being evaluated by fMRI, and found 
more significant activation of the ipsilateral sensorimotor cortex during mirror use than with non-mirrored movement. Bhasin et al. [17] also compared pre-treatment and post-treatment fMRIs in 20 stroke patients receiving 8 weeks of mirror therapy. There were significant improvements in the laterality index (LI) of the ipsilateral primary motor cortex and supplementary/premotor cortex in all patients. They postulated the 'restitution' principle of neural plasticity as the underlying mechanism.

The concept of mirror-induced illusional visual feedback sufficiently explains the way mirror therapy triggers neural plasticity. Mirror therapy on the paretic limb was commonly employed using the following method: place the mirror in the patients' mid-sagittal plane, so that the patient watches the reflected movement of the nonparetic limb as if it were the paretic limb. As a result, it creates a visual illusion of intact movement of the impaired limb. This mirror-made visual illusion facilitates cortical activation and reorganization, thereby augmenting the recovery.

We assumed that the same mechanism could underlie the amelioration of facial muscle weakness after stroke. However, creating illusions of facial movement was a problem. There had been no previous modes of mirror therapy for facial paresis, because placing a mirror in the mid-sagittal plane was impossible for the face. Thus, use of mirror therapy for facial paresis was challenging. We designed a tablet PC application-based mirror therapy to overcome this restriction. Any application converting images from right to left could be applicable. With the application, the patients could watch the intact illusion of the impaired face. As a result, our outcome, effective mirror therapy in facial paresis, was following previous studies with peripheral limbs. Thus, we think it was a meaningful attempt to develop a new rehabilitation method for poststroke facial paresis.

This study has some limitations. First, the sample size was small; hence, larger studies are needed to validate our findings. Second, the results showed minimal changes and large deviations, which might be considered the effect of measurement error. However, to minimize the error, the patients were assessed three times each by two psychiatrists with the same measuring tape. Third, outcome measurements were made by two individuals, one of them being the director of the study; this increases the possibility of observer bias. To decrease this bias, one other physiatrist who did not know which patients were in the study group also measured the outcome, and the mean value was used for data analysis. Fourth, in previous studies, the distance between the corner of the mouth and earlobe was measured only by two-dimensional (2-D) video analysis [2,4]. However, since we thought three-dimensional (3-D) measurement would be much more reliable for the face, we adopted direct measurement of facial movement on the patient's face. After we launched this study, Katsumi et al. [18] introduced a 3-D video analysis to evaluate facial nerve palsy, because 3-D analysis could measure anteroposterior mouth movement, whereas 2-D analysis could not. Although our method was also three dimensional and practical, its validity and reliability was unproven and limited. Finally, we did not use neuroimaging examinations in the study; hence, further study will be needed to elucidate the underlying mechanism.

Despite these limitations, this study has important strengths. From clinical experience, facial paresis is particularly challenging in stroke patients. There are few treatment methods with proven effectiveness; hence, rehabilitation of central facial paresis is limited and difficult. Although mirror therapy has been proven effective for stroke rehabilitation, it had not been applied to central facial paresis, because making an illusion of the face was not feasible. We found a way to overcome this limitation by designing a tablet PC application-based mirror therapy. This study is the first to introduce mirror therapy for post-stroke central facial paresis. We have shown positive outcomes after employing this new rehabilitation method. We expect physiatrists to start using mirror therapy on central facial paresis, and thus a larger study would be possible in the future.

Though this study had several limitations, the results showed that mirror therapy using a tablet PC might be an effective tool for treating central facial paresis after a stroke. This study is challenging, as the first to introduce mirror therapy for central facial paresis. Our sample size was small, so larger studies are needed to confirm the effectiveness of this new rehabilitation method for poststroke facial paresis.

\section{CONFLICT OF INTEREST}

No potential conflict of interest relevant to this article was reported. 


\section{REFERENCES}

1. Pavese C, Cecini M, Camerino N, De Silvestri A, Tinelli C, Bejor M, et al. Functional and social limitations after facial palsy: expanded and independent validation of the Italian version of the facial disability index. Phys Ther 2014;94:1327-36.

2. Konecny P, Elfmark M, Urbanek K. Facial paresis after stroke and its impact on patients' facial movement and mental status. J Rehabil Med 2011;43:73-5.

3. Dafer RM, Rao M, Shareef A, Sharma A. Poststroke depression. Top Stroke Rehabil 2008;15:13-21.

4. Konecny P, Elfmark M, Horak S, Pastucha D, Krobot A, Urbanek K, et al. Central facial paresis and its impact on mimicry, psyche and quality of life in patients after stroke. Biomed Pap Med Fac Univ Palacky Olomouc Czech Repub 2014;158:133-7.

5. Svensson BH, Christiansen LS, Jepsen E. Treatment of central facial nerve paralysis with electromyography biofeedback and taping of cheek: a controlled clinical trial. Ugeskr Laeger 1992;154:3593-6.

6. Nakamura K, Toda N, Sakamaki K, Kashima K, Takeda N. Biofeedback rehabilitation for prevention of synkinesis after facial palsy. Otolaryngol Head Neck Surg 2003;128:539-43.

7. Azuma T, Nakamura K, Takahashi M, Ohyama S, Toda $\mathrm{N}$, Iwasaki $\mathrm{H}$, et al. Mirror biofeedback rehabilitation after administration of single-dose botulinum toxin for treatment of facial synkinesis. Otolaryngol Head Neck Surg 2012;146:40-5.

8. Rossiter HE, Borrelli MR, Borchert RJ, Bradbury D, Ward NS. Cortical mechanisms of mirror therapy after stroke. Neurorehabil Neural Repair 2015;29:444-52.

9. Claflin ES, Krishnan C, Khot SP. Emerging treatments for motor rehabilitation after stroke. Neurohospitalist 2015;5:77-88.

10. Thieme H, Mehrholz J, Pohl M, Behrens J, Dohle C. Mir- ror therapy for improving motor function after stroke. Cochrane Database Syst Rev 2012;(3):CD008449.

11. Reitzen SD, Babb JS, Lalwani AK. Significance and reliability of the House-Brackmann grading system for regional facial nerve function. Otolaryngol Head Neck Surg 2009;140:154-8.

12. Selvaraj S, Stephen R, Paul P, Bright B, Sanjeev MP, Judy AD. Mirror therapy enhances motor performance in the paretic upper limb after stroke: a pilot randomized controlled trial. Arch Phys Med Rehabil 2014;95:2000-5.

13. Kim H, Shim J. Investigation of the effects of mirror therapy on the upper extremity functions of stroke patients using the manual function test. J Phys Ther Sci 2015;27:227-9.

14. Colomer C, NOe E, Llorens R. Mirror therapy in chronic stroke survivors with severely impaired upper limb function: a randomized controlled trial. Eur J Phys Rehabil Med 2016;52:271-8.

15. Sutbeyaz S, Yavuzer G, Sezer N, Koseoglu BF. Mirror therapy enhances lower-extremity motor recovery and motor functioning after stroke: a randomized controlled trial. Arch Phys Med Rehabil 2007;88:555-9. 16. Guo F, Xu Q, Abo Salem HM, Yao Y, Lou J, Huang X. The neuronal correlates of mirror therapy: a functional magnetic resonance imaging study on mirrorinduced visual illusions of ankle movements. Brain Res 2016;1639:186-93.

17. Bhasin A, Padma Srivastava MV, Kumaran SS, Bhatia $\mathrm{R}$, Mohanty S. Neural interface of mirror therapy in chronic stroke patients: a functional magnetic resonance imaging study. Neurol India 2012;60:570-6.

18. Katsumi S, Esaki S, Hattori K, Yamano K, Umezaki T, Murakami S. Quantitative analysis of facial palsy using a three-dimensional facial motion measurement system. Auris Nasus Larynx 2015;42:275-83. 